\title{
Dynamics of lipids in synthetic membranes
}

\author{
Robert A. Moss \\ Department of Chemistry, Rutgers, The State University of New Jersey, New \\ Brunswick, New Jersey 08903
}

\begin{abstract}
Bilayer coliposomes were created from blends of head-group functionalized and non-functionalized synthetic lipids. Functionalized lipids in the outer leaflets of the bilayers were then chemically differentiated from the (unchanged) functionalized lipids of the inner leaflets, thus permitting study of the dynamics of the ensuing reequilibration that occurred via thermally activated transbilayer lipid migration ("flip-flop"). Our particular focus was the relation between lipid molecular structure and flip-flop dynamics within the bilayer membranes. The structural factors investigated included chain length, unsaturation, cyclopropanation, alkylation, and "stiffening". The effects of head group type (ammonium ion or phosphocholine) and overall lipid character (monopolar or bolaamphiphilic) were also examined. The results highlight factors that simultaneously allow both the membrane fluidity and maintenance of leaflet differentation that are essential to proper biological function.
\end{abstract}

Synthetic bilayer lipid vesicles or liposomes are of interest in their own right and as models for biological membranes (ref. 1,2). These closed, hollow spheres or ellipsoids create aqueous reaction loci of different chemical or microenvironmental properties separated by hydrocarbon barriers. Thus the permeation of reagents and ions across these barriers can be controlled, and their reactivity modulated. Moreover, one can chemically differentiate the exoliposomal and endoliposomal surfaces, furthering the analogy to biological membranes, where transverse asymmetry between the exo and endo leaflets of the bilayer is common and functionally significant (ref. 1-5). With differentiated bilayer surfaces, we can study the dynamics of individual lipid molecules as they undergo reequilibration, and we can relate the dynamics to the lipid's molecular structure.

The most common biological lipids are phosphoglycerides, e.g. 1. Kunitake (ref. 6) and

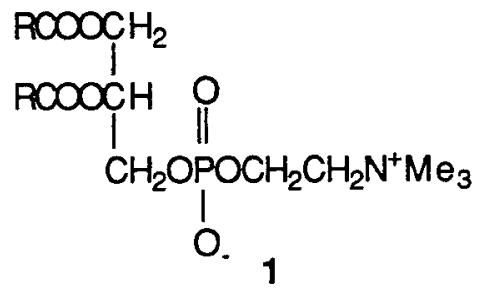

$$
\left(n-\mathrm{C}_{18} \mathrm{H}_{37}\right)_{2} \mathrm{~N}^{+} \mathrm{Me}_{2}, \mathrm{Cl}^{-}
$$

Fendler (ref. 7) showed that double chain quaternary ammonium ions (2) could also form liposomes, so that one could model bilayer membranes with lipids of the simplest structure. 
Moreover, we could envision a rational sequence of lipid molecular structures that gradually increased in complexity, incrementally bridged the gulf between 2 and 1, and traced the relation between lipid structure and lipid dynamics at the aggregate or liposomal level.

\section{SURFACE DIFFERENTIATION OF LIPOSOMES}

The generation of surface differentiated liposomes is a crucial element in the modeling of biological membranes (ref. 8-10). Our methodology chemically differentiates exo from endo liposomal head groups of functionalized lipids by reactions that preferentially occur at the bilayer's exoliposomal surface.

For example, 1:9 coliposomes of functional (F) (3-F) and non-functional (NF) (2) lipids were created by sonication at pH 3.9 (ref. 11). These liposomes had a gel to liquid crystalline phase transition temperature $\left(I_{c}\right)$ of $35.5^{\circ} \mathrm{C}$, where the phase change is associated with conversion of the all s-trans, staggered conformer, well-packed lipid chains of the relatively rigid, non-leaky, lower temperature gel phase liposomes to the looser, leakier, and "fluid" liquid-crystalline liposomes which feature gauche conformers or "kinks" in their chains (ref. 1-3).

The 3-F/2 coliposomes were surface differentiated by brief exposure to glutathione at an external $\mathrm{pH}$ of 7.9 , thus rapidly cleaving the exoliposomal p-nitrophenyl benzoate residues (G) of 3-F to the corresponding $\mathbf{p}$-nitrophenylate moieties ( $\left.\mathbf{G}^{\prime}\right)$. This process was monitored at $400 \mathrm{~nm}$ and occurred with a "fast" rate constant, $\mathrm{k}_{f}=0.11 \mathrm{~s}^{-1}$.<smiles>O=C(Oc1ccccc1)c1ccccc1[N+](=O)[O-]</smiles>

Subsequent, slower $\left(k_{S}=7.5 \times 10^{-4} s^{-1}\right)$ endoliposomal cleavage of $G$ was rate limited by trans-membrane $\mathrm{H}^{+} / \mathrm{OH}^{-}$(actually concurrent counterion) permeation driven by the imposed $\mathrm{pH} 7.9 / 3.9$ gradient (ref. 12). Counterion $\left(\mathrm{Na}^{+}\right.$or $\mathrm{K}^{+}$) permeation was slowed by the liposomal bilayer's cationic lipids; the $\mathrm{pH}$ gradient rapidly collapsed if valinomycin was added to "ferry" the metal cations (ref. 13).

The biphasic absorption changes attending the exo/endo cleavage of the 3-F/2 coliposomes are illustrated in Figure 1. After the initial, exoliposomal cleavage $(\sim 1 \mathrm{~min})$, the exo surfaces bear $\mathrm{ArO}^{-}\left(\mathbf{G}^{\prime}\right)$ residues at $\mathrm{pH} 8$, while the endo liposomal membranes still carry intact ester groups at pH 3.9. We then monitor endo to exo transverse bilayer migration ("flip-flop") of the functional lipids by the following protocol (ref. 11). (1) After exoliposomal cleavage by $\mathrm{RS}^{-}$at $\mathrm{pH} 8$, the external $\mathrm{pH}$ is reduced to 3.9 with $\mathrm{HCl}$, quenching further esterolysis or $\mathrm{H}^{+} / \mathrm{OH}^{-}$permeation. (2) The now surface-differentiated liposomes are incubated at a preselected temperature (e.g. $\geq I_{C}$ ) for a variable time period. (3) The liposomes are cooled to $25^{\circ} \mathrm{C}$. (4) The $\mathrm{pH}$ is readjusted to 7.9 with $\mathrm{NaOH}$ and esterolysis is monitored at $400 \mathrm{~nm}$. Figure 2 illustrates typical results.

The key observation is the appearance of a new fast cleavage induced by the incubation. In the absence of the heating step, we observe only the normal biphasic behavior shown in Figure 1; the dotted line in Figure 2 shows a control experiment in which heating is omitted. In constrast, the heating process (solid line) bifurcates the original, slow, endoliposomal 


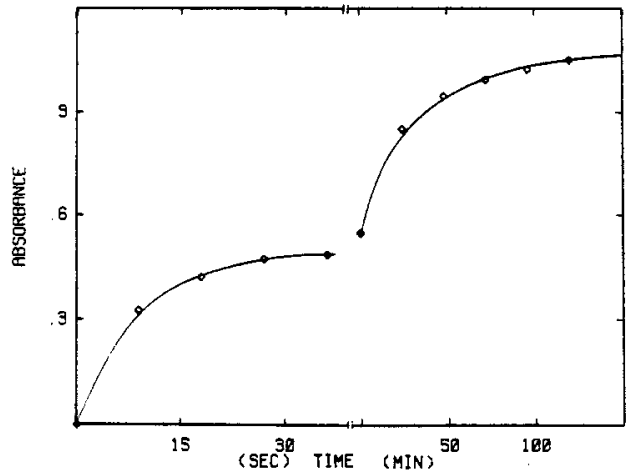

Figure 1. Absorbance at $400 \mathrm{~nm}$ vs. time for the appearance of $\mathrm{p}$-nitrophenylate from coliposomal 3-F/2 at an external pH of 7.9

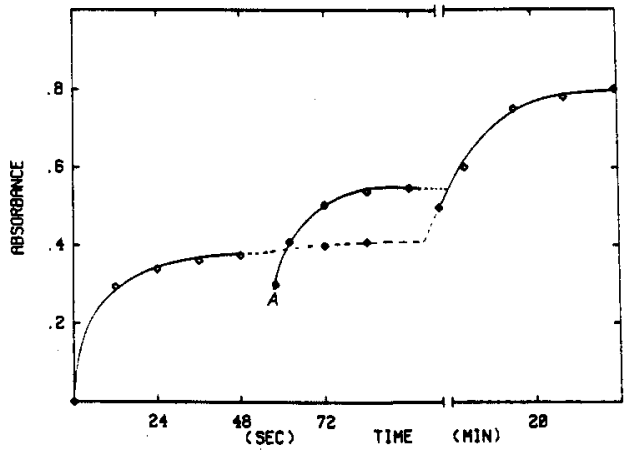

Figure 2. Same as Figure 1, but with the imposition of the flip-flop protocol described in the text.

cleavage into 2 parts, a new fast phase and a residual slow phase. The new $\mathrm{kf}_{\mathrm{f}}$ represents $15 \%$ of cleavage after $1 \mathrm{~min}$ at $40^{\circ} \mathrm{C}, 20 \%$ after $2 \mathrm{~min}$, and $28 \%$ after 6 or $12 \mathrm{~min}$ of heating. These results represent those intact 3-F lipids that flipped from endo to exo locations during incubation. After $6 \mathrm{~min}$, the original $\underline{k}_{S}$ is replaced by equal contributions of $\mathrm{k}_{f}$ and $\mathrm{k}_{S}$; the endoliposomal 3-F lipids have been equilibrated between endo and exo sites.

Note that the new fast cleavage begins below the end absorbance of the initial fast cleavage (Fig. 2, point A), corresponding to cleaved (G') exo 3-F lipids that "flopped" to endo sites during incubation. Their $\mathbf{G}^{\prime}$ residues protonated to $\mathrm{p}$-nitrophenols under the $\mathrm{pH} 3.9$ endoliposomal conditions and lost absorbance at $400 \mathrm{~nm}$.

The half-time $\left(t_{1} / 2\right)$ for reequilibration of the surface-differentiated 3-F/2 coliposomes is only $\sim 2 \mathrm{~min}$ at $40^{\circ} \mathrm{C}$, whereas $t_{1 / 2}$ for dipalmitoylphosphatidylcholine $\left(1, \mathrm{R}=\mathrm{C}_{15} \mathrm{H}_{31}\right)$ liposomes is $-6.5 \mathrm{~h}$ at $30^{\circ} \mathrm{C}$ (ref. 14). The disparity, reflecting the simpler molecular structure of $3-\mathrm{F}$ as well as its cationic head groups, will be discussed below.

\section{LIPID ARCHITECTURE AND DYNAMICS}

\section{Chain length and backbone}

With the surface differentiation and flip-flop protocols we can determine how variations in the molecular structures of functional lipids affect their dynamic behavior within liposomal membranes. A simple application examines the dependence on chain length for homologues of 3-F and 2.

With $\mathrm{R}=\mathrm{n}-\mathrm{C}_{16} \mathrm{H}_{33}, \mathrm{n}-\mathrm{C}_{18} \mathrm{H}_{37}$, or $\mathrm{n}-\mathrm{C}_{20} \mathrm{H}_{41}, 1: 10 \mathrm{~F} / \mathrm{NF}$ coliposomes had $\mathrm{I}_{c}$ 's of 26,39 or $48^{\circ}$ C. The corresponding surface-differentiated liposomes exhibited flip-flop dynamics determined by their $I_{c}$ 's; i.e., by their gel to liquid crystalline transitions (ref. 15). The $C_{16}$ coliposomes (surface differentiated at $15^{\circ} \mathrm{C}$ ) reequilibrated in $\sim 1$ min at $25^{\circ} \mathrm{C}$; the $\mathrm{C}_{18}$ coliposomes were stable to flip-flop for $>1 \mathrm{~h}$ at $25^{\circ} \mathrm{C}$ (below $I_{c}$ ) but reequilibrated in $\sim 1 \mathrm{~min}$ at $38-40^{\circ} \mathrm{C}$. Finally, the $\mathrm{C}_{20}$ coliposomes were stable at $40^{\circ} \mathrm{C}$, but rapidly reequilbrated at 50 ${ }^{\circ} \mathrm{C}$ (ref. 15). Liposomes constructed of simple ammonium ions thus display flip-flop that is governed by chain packing: below $I_{c}$ the well-packed gel phase lipids resist flip-flop, above $I_{c}$ the more fluid, liquid crystalline lipids rapidly reequilbrate between the endo and exoliposomal leaflets. 


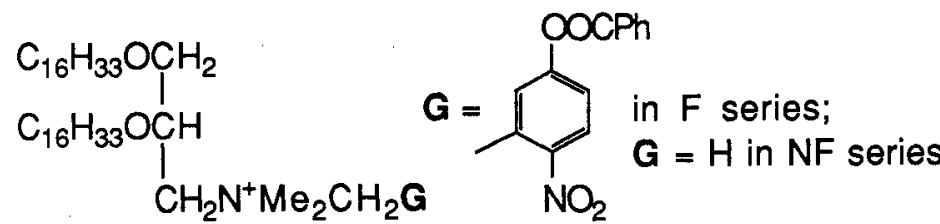

4

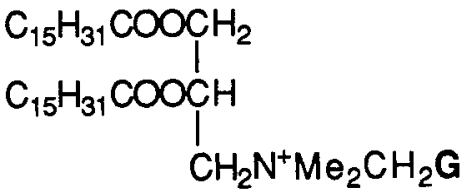

5

Greater molecular complexity confers additional stability. Lipids 4 and 5 feature 3-carbon pseudogylceryl backbones with ether (4) or ester (5) linkages to 16-carbon chains at $C_{1}$ and $\mathrm{C}_{2} ; \mathrm{C}_{3}$ carries head groups analogous to those of 2 (NF, G $=\mathrm{N}^{+} \mathrm{Me} \mathrm{e}_{3}$ ) or $3(\mathrm{~F}, \mathrm{G}=\mathrm{p}$ nitrophenyl benzoate) (ref. 16). The $I_{C}$ 's of $1: 7 \mathrm{~F} / \mathrm{NF}$ coliposomes are $37^{\circ} \mathrm{C}(4)$ and $44^{\circ} \mathrm{C}(5)$. Surface differentiated coliposomes of 4 reequilibrate with $t_{1 / 2} \sim 5 \mathrm{~min}$ at $40^{\circ} \mathrm{C}$ and $1 \mathrm{~min}$ at $45^{\circ}$ $\mathrm{C}$, whereas the ester coliposomes of 5 display $\mathrm{t}_{1 / 2} \sim 5 \mathrm{~min}$ at $55^{\circ} \mathrm{C}$ and $\sim 1 \mathrm{~min}$ at $65^{\circ} \mathrm{C}$. Both 4 and 5 , in contrast to 3 , display some resistance to flip-flop above their $I_{c}$ 's, probably reflecting better chain packing from their vicinal double long chain pseudoglyceryl architecture.

\section{Phytanyl lipids and bolaamphiphiles}

Unusual lipids (6) were isolated from thermophylic archaebacteria that thrive at high $\left(50-80^{\circ}\right.$ C) temperatures (ref. 17). These phytanyl isoprenoid lipids feature ether linkages to glyceryl backbones with phosphoethanolamine head groups. They are "double-headed" or bolaamphiphiles, capable when extended of forming a monolayer liposomal membrane (note the 32-carbon main chain of 6).

A simple model for 6 is the 1,2-diphantanyl pseudoglycerylammonium lipid, 7 (ref. 18), but compared to 1:10 F/NF coliposomes of straight chain ether lipid 4, phytanyl coliposomes of 7

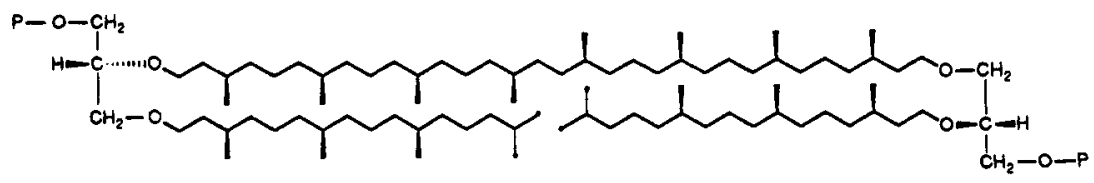

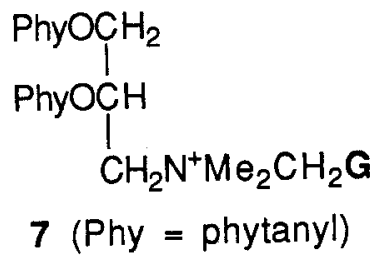

are very floppy. The $I_{c}$ of 7 is $<10^{\circ} \mathrm{C}$, and the phytanyl coliposomes (surface-differentiated at $5^{\circ} \mathrm{C}$ ) reequilibrate with $t_{1 / 2}<1 \mathrm{~min}$ at $12^{\circ} \mathrm{C}$. Chain branching and interchain $\mathrm{Me} / \mathrm{Me}$ interactions must reduce the stability of coliposomal 7 relative to that of $4\left(\mathrm{t}_{1 / 2}>10 \mathrm{~min}\right.$ at $25^{\circ}$ C) (ref. 17-19).

A better model for 6 is the desmethylated, pseudoglyceryl functional bolaamphiphile 8-F (ref. 20). Coliposomes of 1:14 8-F in nonfunctional host 4 , when surface-differentiated, reveal a 70:30 exoliposomal $\left(k_{f}=0.14 \mathrm{~s}^{-1}\right)$ to endoliposomal $\left(k_{s}=5.8 \times 10^{-4} \mathrm{~s}^{-1}\right)$ kinetic partition of the 
8-F esterolysis. This excludes coliposomal arrangements in which the 8-F lipids assume only extended, bilayer bridging conformations that extend across both leaflets of the bilayer. Such a situation would demand a 50:50 exo/endo kinetic partition. The observations are consistent with at least $40 \%$ of the 8-F lipids adopting U-plan, bent conformations that concentrate their head groups in the outer bilayer leaflet, where, relative to the endo leaflet, greater surface area is available due to the gentler curvature. Similar U-plan bolaamphiphile conformations are seen in air/water monolayers (ref. 17).

After surface differentiation of the 8-F/4-NF coliposomes, endoliposomal 8-F head groups undergo facile flip-flop $\left(\mathrm{t}_{1 / 2} \sim 5 \mathrm{~min}\right.$ at $25^{\circ} \mathrm{C}$ ) at a rate similar to that of the host lipid molecules (t $1 / 2 \sim 5 \mathrm{~min}$ at $40^{\circ} \mathrm{C}$ for 4-F/4-NF coliposomes) (ref. 20). Although 8-F fails to manifest exclusive bilayer bridging and resitance to flip-flop, these desirable properties can be evoked if we "stiffen" the main chain with a biphenyl unit; cf. 9-F. Now U-plan conformations are inhibited and the bolaamphiphile extends across the bilayer. Exoliposomal/endoliposomal surface differentiation exhibits a 50:50 partition, with very slow flip-flop of the extended 9-F lipids (ref. 21); we observe only $\sim 11 \%$ reequilibration after $1 \mathrm{~h}$ at $50^{\circ} \mathrm{C}$, reflecting the difficulty

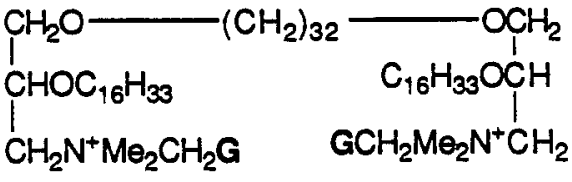

8-F

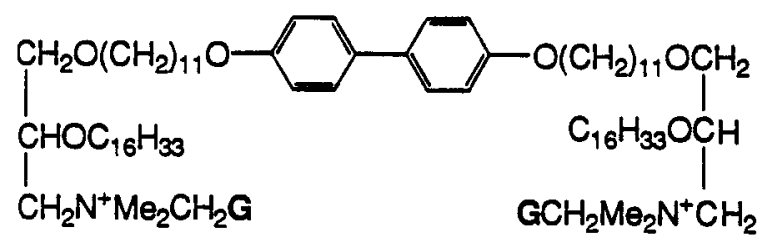

9-F

in bending biphenyl-stiffened 9-F within the bilayer. Thus extended 9-F lipids maintain their bilayer-bridging arrangement at temperatures that greatly exceed the $I_{c}$ of the host-bilayer. This should allow appropriate analogues to serve as reporter probes during the interconversions of gel and liquid crystalline host bilayers.

\section{Unsaturated and cyclopropanated lipids}

A carbon-carbon double bond in a lipid's hydrocarbon chain creates a permanent "kink" that disturbs chain packing, lowers the $I_{C}$, and fosters fluidity within the liposome (ref. 1-3). Unsaturated lipids figure prominently in biological membranes, so we prepared the bis- $\mathrm{C}_{18}$ pseudoglyceryl model lipids 10 (stearoyl) and 11 (cis-11, oleoyl; trans-11, elaidoyl) (ref. 22).

The contrast in $I_{C}$ 's of their liposomes is instructive; liposomes of saturated 10 have $I_{C}=59$ ${ }^{\circ} \mathrm{C}$, whereas those of trans-11 and cis-11 display depressed $I_{C}$ 's of $14^{\circ} \mathrm{C}$ and $<10^{\circ} \mathrm{C}$. The unsaturation-induced fluidity is expressed in the liposomal dynamics where 1:10 F/NF surface differentiated coliposomes of 10 stoutly resist flip-flop below their $I_{C}$ ( $t_{1 / 2}>30 \mathrm{~min}$ at $40^{\circ} \mathrm{C}$ ), while coliposomes of cis- or trans-11 undergo rapid flip-flop with $t_{1 / 2} \sim 3$ or $8 \mathrm{~min}$, respectively, at $15^{\circ} \mathrm{C}$ (ref. 22). The greater floppiness of the cis-11 liposomes reflects the greater disturbance to chain packing created by a cis vs. a trans double bond (ref. 1-3).

The supressed $I_{c}$ and decreased resistance to flip-flop is consistent with previous reports of looser chain packing in liposomes constructed from unsaturated lipids (ref. 23), but our cationic lipids appear more susceptible to flip-flop at ambient temperature than related, zwitterionic phospholipids; dioleoylphosphatidylchloline liposomes are reported to have a $t_{1} / 2$ of several days at $30^{\circ} \mathrm{C}$ (ref. 24). The difference must be related to the differing head groups (see below). 


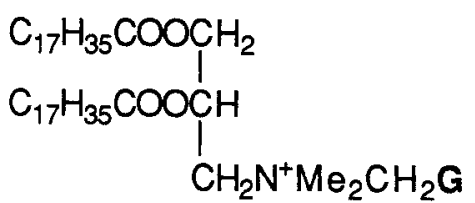

10

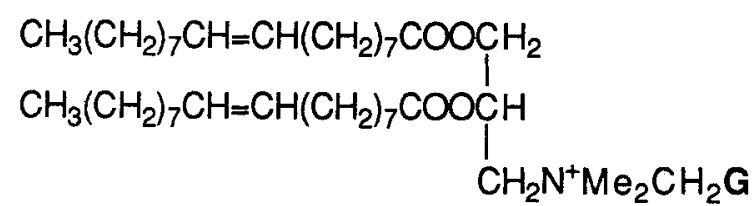

11

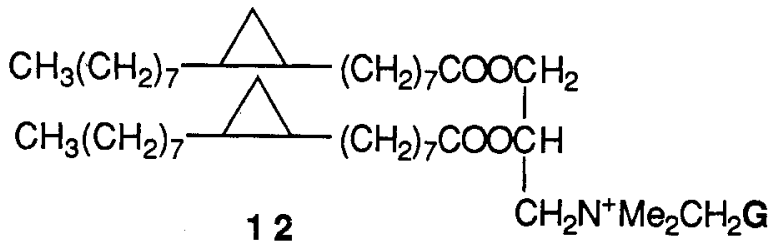

Certain bacteria and protozoa possess phospholipids bearing cyclopropanated chains derived from dihydrosterculic acid. These afford bilayers that are less fluid and more ordered at ambient temperatures than bilayers composed of analogous unsaturated lipids (ref. 25). The cyclopropyl group induces a low $I_{C}$ (like $C=C$ ), but it also provides a barrier to the transmission of segmental motion along the chain, imposing greater ordering relative to the unsaturated chain. High local ordering and long motional correlation times might fit the cyclopropyl lipids for a biological regulatory role in the dynamic processes that affect membrane stability.

We prepared the bis-cyclopropanated pseudoglyceryl ammonium ion lipid, 12, for comparison to bis-oleoyl lipid cis-11 (ref. 26, 22). The small (450 A) 1:10 F/NF coliposomes of dihydrosterculoyl 12 displayed a low $I_{C}\left(<10^{\circ} \mathrm{C}\right)$, similar to that of oleoyl liposomes of 11, but the surface differentiated cyclopropanated liposomes reequilibrated more slowly: $t_{1 / 2} \sim 16$ min at $25^{\circ} \mathrm{C}$, compared to $t_{1 / 2}<1 \mathrm{~min}$ for the analogous cis- -11 liposomes at the same temperature (ref. 26). The origins of this disparity can be traced to the lipids' conformational states. Fourier transform IR revealed fewer disruptive double gauche conformational sites in the acyl chains of 12 than in those of cis-11. This can be associated with better chain packing, increased chain order, and greater resistance to flip-flop in lipsomal 12. Though dynamically less stable than such saturated analogues as 5 or 10, cyclopropanated liposomes of 12 are indeed more stable than their unsaturated analogues.

\section{Saturated and unsaturated phospholipids}

Pseudoglycerylammonium ion liposomes are relatively unstable toward flip-flop; above their $I_{c}$, the fluid, liquid crystalline liposomes are subject to facile trans-bilayer lipid migration. The cationic head groups of these lipids are mutually repulsive, so that their positional stability within the bilayer must depend entirely upon the packing of their long chains. Any factor that degrades close packing will render these lipids fluid and flippable.

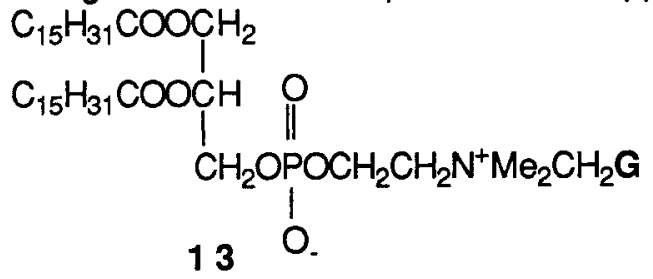

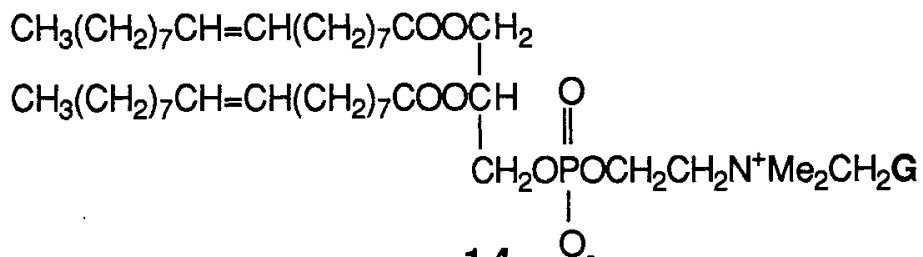


Biological membranes contain unsaturated lipids and are generally fluid, as required for facile passage of essential molecular traffic. Yet these membranes retain surface differentiation, including a transverse asymmetry of lipid distribution (ref. 1-5). Their maintenance of bilayer fluidity and surface differentiation must be a function of their head groups, which are often zwitterionic $(e, a, 1)$, and can be mutally attractive, in contrast to the cationic head groups of synthetic ammonium lipids like 5.

To test these ideas, we compared coliposomes constructed from saturated and unsaturated ammonium ion lipids 5(10) and 11 with saturated and unsaturated phosphatidylcholines 13 and 14 (ref. 13). Coliposomes were prepared from 1:7 or 1:10 F/NF lipid blends, were small $(d=310-420 \AA)$, and unilamellar. The $I_{c}$ 's of the saturated liposomes are similar $\left(5,44^{\circ} \mathrm{C}\right.$ and $13,40^{\circ} \mathrm{C}$ ), but the surface differentiated phospholipids are more resistant to flip-flop than the ammonium ion lipids: approximate $t_{1 / 2}$ values at $60^{\circ} \mathrm{C}$ are $16 \mathrm{~min}$ for 13 and $2 \mathrm{~min}$ for 5 (ref. 13). [Note that these reequilibrations are conducted above the $I_{c}$ 's; at $25^{\circ} \mathrm{C}$ the reequilibration of either liposome would require hours.]

The enhanced stability of the phospholipids becomes dramatically clear when chain unsaturation is introduced. As we have seen (ref. 22), liposomes of unsaturated pseudoglycerylammonium ions (e.g. cis-9) feature low $I_{C}$ 's, and are fluid and floppy: $t_{1 / 2} \sim 3$ min at $15^{\circ} \mathrm{C}$. In contrast, no diminution in resistance to flip-flop attends unsaturation in phospholipids. As expected, the $I_{C}$ drops sharply with unsaturation $\left(40^{\circ} \mathrm{C}\right.$ for dipalmitoyl liposomal 13 vs. $\angle 0^{\circ} \mathrm{C}$ for dioleoyl 14), insuring bilayer fluidity at ambient or physiological temperatures. However, stability toward reequilibration of the surface differentiated, unsaturated phospholipids is actually enhanced: $t_{1 / 2} \sim 6 \mathrm{~min}$ at $65^{\circ} \mathrm{C}$ for 13 , but $t_{1 / 2} \sim 20 \mathrm{~min}$ at $65^{\circ} \mathrm{C}$ for 14 (ref. 13).

Thus, bilayers constructed of unsaturated, zwitterionic phosphatidylcholines retain their resistance to flip-flop far above their $I_{C}$. Indeed, an Arrhenius study yields an activation energy of $\sim 24 \mathrm{kcal} / \mathrm{mol}$ for the reequilibration of liposomal 14 , affording $t_{1} / 2 \sim 24 \mathrm{~h}$ at $30^{\circ} \mathrm{C}$ by extrapolation. This is in order of magnitude agreement with McConnell's value of $t_{1 / 2} \sim 2.6-7$ $\mathrm{h}$ at $30^{\circ} \mathrm{C}$ for a spin-labeled dipalmitoylphosphatidylcholine probe in egg lecithin liposomes (ref. 14). It seems possible that $\pm / \pm$ attractive electrostatic interactions may oppose flip-flop with 14 or 13, even when their bilayers are in the fluid state (ref. 27).

In dynamic terms, coliposomes containing functional lipids 13 or 14 are good models for biologically relevant bilayer membranes. Our investigations can now turn toward the effects of additives such as cholesterol, peptides and proteins, or sphingomyelin on membrane permeability and stability. Most recently, we have shown that mechanical stress created by thermally responsive polymers bound to the exteriors of differentiated liposomes of 13 can "catalyze" endo to exoliposomal flip-flop, presumably by generating defects or "holes" in the exoliposomal leaflets (ref. 28).

\section{Acknowledaments}

I am very grateful to my colleagues and collaborators, whose names appear in the references. All of us thank the U.S. Army Research Office for its support of this research program.

\section{REFERENCES}

1. R.B. Gennis, Biomembranes: Molecular Structure and Function, Springer, New York (1989).

2. M.K. Jain, Introduction to Biological Membranes, 2nd Ed., Wiley, New York (1988).

3. J.H. Fendler, Membrane Mimetic Chemistry, Wiley, New York (1982). 
4. A. Zachowski and P.F. Devaux, Comments Mol. Cell. Biophys, 6,63 (1989).

5. J.A.F. Op den Kamp, Annu. Rev. Biochem., 48, 47 (1979).

6. T. Kunitake and Y. Okahata, J. Am. Chem. Soc., 99, 3860 (1977).

7. C.D. Tran, P.L. Klahn, A. Romero, and J.H. Fendler, J.Am. Chem. Soc., 100, 1622 (1978).

8. J.-H. Fuhrhop and J. Mathieu, Angew. Chem. Int. Ed. Engl. 23, 100 (1984).

9. J.-H. Fuhrhop and D. Fritsch, Acc. Chem. Res., 19, 130 (1986).

10. H. Ringsdorf, B. Schlarb, and J. Venzmer, Angew. Chem. Int. Ed. Engl, 27, 113 (1988).

11. R.A. Moss, S. Bhattacharya, and S. Chatterjee, J. Am. Chem. Soc., 111, 3680 (1989).

12. R.A. Moss and T. Fujita, Tetrahedron Lett, 31, 2377 (1990).

13. R.A. Moss and Y. Okumura, J. Am. Chem. Soc., 114, 1750 (1992).

14. R. Kornberg and H.M. McConnell, Biochemistry, 10, 1111 (1971). M.G. McNamee and H.M. McConnell, Biochemistry, 12, 2951 (1973).

15. R.A. Moss, T. Fujita, and S. Ganguli, Langmuir, 6 , 1197 (1990).

16. R.A. Moss, S. Ganguli, Y. Okumura, and T. Fujita, J.Am. Chem. Soc. 112, 6391 (1990).

17. K. Yamauchi, Y. Sakamoto, A. Moriya, K. Yamada, T. Hosokawa, T. Higuchi and M. Kinoshita, J.Am. Chem. Soc. 112, 3188 (1990). L.C. Stewart, M. Kates, I.H. Ekiel, and C.P. Smith, Chem. Phys. Lipids, 54, 115 (1990). K. Yamauchi, A. Moriya, and M. Kinoshita, Biochim. Biophys. Acta, 1003, 151 (1989).

18. R.A. Moss and T. Fujita, Tetrahedron Lett. 31,7559 (1990).

19. F.M. Menger, M.G. Wood, Jr., Q.Z. Zhou, H.P. Hopkins, and J. Fumero, J. Am. Chem. Soc., 110, 6804 (1988). F.M. Menger, M.G. Wood, Jr., S. Richardson, Q. Zhou, A.R. Elrington, and M.J. Sherrod, J.Am. Chem. Soc., 110, 6797 (1988).

20. R.A. Moss, T. Fujita, and Y. Okumura, Langmuir, 7, 2415 (1991).

21. R.A. Moss and J-M. Li, J. Am. Chem. Soc. 114, 9227 (1992).

22. R.A. Moss, T. Fujita, and Y. Okumura, Langmuir, 7, 400 (1991).

23. P.J. Quinn, F. Joo, and L. Vigl, Proo. Biophys. Mol. Biol., 53, 71 (1989).

24. B. de Kruijff and K.W.A. Wirtz, Biochim. Biophy. Acta, 468, 318 (1977).

25. E.J. Dufourc, I.C.P. Smith, and H.C. Jarrell, Chem. Phys. Lipids, 33, 153 (1983). E.J. Dufourc, I.C.P. Smith, and H.C. Jarrell, Biochemistry, 23, 2300 (1984). H.C. Jarrell, A.P. Tulloch, and I.C.P. Smith, Biochemistry, 22, 5611 (1983).

26. R.A. Moss, T. Fujita, Y. Okumura, Z. Hua, R. Mendelsohn, and L. Senak, Langmuir, 8, 1731 (1992).

27. P.G. Scherer and J. Seelig, Biochemistry, 28, 7720 (1989). J. Seelig, P.M. Macdonald, and P.G. Scherer, Biochemistry, 26, 7535 (1987).

28. S. Bhattacharya, R.A. Moss, H. Ringsdorf, and J. Simon, J.Am. Chem. Soc., 115, 3812 (1993). 